\title{
From Quasars to Extraordinary N-body Problems
}

\author{
D. Lynden-Bell
}

Clare College, Cambridge \& Institute of Astronomy. Visiting Professorial Fellow, The Queen's University, Belfast.

\begin{abstract}
We outline reasoning that led to the current theory of quasars and look at George Contopoulos's place in the long history of the N-body problem. Following Newton we find new exactly soluble N-body problems with multibody forces and give a strange eternally pulsating system that in its other degrees of freedom reaches statistical equilibrium.
\end{abstract}

\section{Introduction}

This meeting is held to honour George Contopoulos for his great contributions to dynamical systems theory and the N-body problem. I shall pay my tribute to him in three parts,

I Placing his contributions in the proud history of those who have made major contributions to the N-body problem.

II Since nothing but the best is good enough to honour George I present to him a copy of my best paper (Lynden-Bell, 1969) and include here a résumé of its arguments that led to the current theory of quasars. There are questions my paper raised 29 years ago which are still unexplored.

III With Prof. Ruth Lynden-Bell (my wife) I present our new extraordinary N-body problems which we solve for all initial conditions. These problems can also be solved in quantum mechanics when the hyper-keplerian potential energy is

$$
V=-N \widetilde{Z} e^{2} / r
$$

where

$$
\begin{gathered}
r^{2}=\sum_{i=1}^{N} \frac{m_{i}}{M}\left(\mathbf{x}_{i}-\overline{\mathbf{x}}\right)^{2}, \\
M=\sum m_{i}
\end{gathered}
$$


i.e., $r$ is the mass-weighted-root-mean-square radius of the N-body system. I quote here the energy and degeneracy of the $n$th quantum state but we shall publish derivations elsewhere.

$$
E_{n}=-\frac{2 M \hbar^{-2}\left(N \widetilde{Z} e^{2}\right)^{2}}{[2 n+3(N-2)]^{2}}
$$

the degeneracy of this $N$ particle state is

$$
g(n, N)=\frac{[n+3(N-2)] !}{(n-1) !(3 N-4) !}[2 n+3(N-2)],
$$

for $N=2, g$ reduces to $n^{2}$ and the energy reduces that of the hydrogen atom for which $M=m_{p}+m_{e}$. To see this, $\widetilde{Z}$ is replaced by $\frac{1}{2}\left(m_{p} m_{e}\right)^{1 / 2} / M$ when $r$ is replaced by the separation of the electron from the proton. We have written the coefficient of the potential energy in the clumsy form $N \widetilde{Z} e^{2}$ so that the analogy to hydrogenic atoms can be readily seen by physicists.

\section{Contributions to the N-body problem (excluding Agatha Christie's)}

The N-body problem probably started with Newton although Hooke would undoubtedly dispute it since he seems to have conceived the idea independently but had not the mathematical ability to work out its consequences. As Chandrasekhar (1996) has shown, Newton's Principia (Newton 1687, Cajori 1934) has much to teach us even today (see Lynden-Bell \& Nouri-Zonoz, 1998). Recent studies of the Portsmouth papers have shown that Newton developed most of the perturbation theory that was hitherto attributed to the mathematical astronomers of the 18th and 19th centuries. Newton's method was to store up the momentum generated by perturbations and then deliver it as an impulse that changed the motion from one ellipse to another. This of course gives him the equations for the variations of the orbital elements which are the meat of perturbation theory. My brief résumé of the N-body problem's history is:

Newton 1687

Laplace 1795

Poincaré 1892-99

Whittaker 1913 (1959)

Contopoulos 1956-

Kolmagorov-Arnold-Moser
Orbit Theory and the general solution of the first extraordinary N-body problem

Perturbation Theory for near circular orbits

Topological Methods

Adelphic Integrals as series

Third Integrals and Chaos

Invariant Tori \& Arnold diffusion 
To these theoretical studies we must add the numerical computation of the Nbody problem and here Aarseth's name stands out as a persistent pioneer exploring this problem (Aarseth, 1974) although many others have contributed, especially Heggie (1975) through his work on triple interactions. Both in globular cluster theory and in dynamical systems Henon's work stands out for its beauty (Henon, 1961, 1969, 1974) while Antonov (1962) was responsible for a fundamental advance in the understanding of gravitational thermodynamics later popularised and extended to negative specific heats and gravitational phase transitions by the author (Lynden-Bell \& Wood, 1968; Lynden-Bell \& Lynden-Bell, 1977)and by Thirring (1972). Betteweiser \& Sugimoto (1984) were responsible for giving the gravothermal instability a delightful new twist in their discovery of the inverse gravothermal catastrophe that leads to giant thermal oscillations. But let me return to what George Contopoulos taught me at our many contacts since 1961.

To set the scene I had written my thesis in 1960 which contained a new derivation of what potentials had local first integrals of the motion besides the energy and the angular momentum about the axis. The main part of the work was the derivation of these different classes of potential while other parts of the thesis contained the time dependent evolution of accretion disks gravitational instability to make a theory of the spiral structure of galaxies. The beliefs of those times are well illustrated by the first edition of Landau \& Lifshitz's book on classical mechanics; either a dynamical system was separable and integrable or it was ergodic (by which was meant that almost all orbits visited all volumes of the phase space accessible under the energy constraint). Having classified the special forms of potential that had local integrals I expected that most other potentials would show ergodic behaviour. From the inequality of the $z$ and $R$ dispersions of the stars in the Galaxy it was clear that there must be another integral other than $E$ and $h$ for the Milky Way so I had begun trying to fit Eddington (1915) (now called Stakle) potentials to galaxies. It was quite shattering when at the 1961 IAU general assembly in Berkeley, George Contopoulos (1960) showed that orbits in most smooth potentials behaved as though there were third integrals. Suddenly the special interest of the special potentials fell away - they were not the only systems with 3rd integrals, merely those for which we knew the exact analytical form of those integrals. They seemed now to be mathematical curiosities rather than systems fundamental to the dynamics of real galaxies.

Three years later George organised a very instructive IAU symposium (No.25) at Thessaloniki on the Theory of Orbits in the Solar System and Stellar Systems. Here he brought into contact the celestial mechanics fraternity, with their long history of analytically calculating orbits in the solar system by perturbation theory, with us new boys who were attempting to understand the statistics of the orbits in the more complicated potentials of galaxies; George Contopoulos $(1965,1966)$ here taught us that many of the problems were common to both fields and showed how fertile it was to bring different communities who knew different things to the same conference - his wide interests have made him especially good at that throughout his life and

\footnotetext{
${ }^{1}$ they got that name only later
} 
this 1998 conference is no exception.

For brevity, I shall skip contacts at Besançon on the N-body problem where George presented Poisson Bracket series for third integrals and we were introduced to Lie series.

In 1973 at Saas Fée George gave lectures in which he introduced me to the wonders of modern dynamical theory - topological methods incomplete chaos and the KAM theorem. It opened my eyes to so much that was new to me that I retreated back to more directly astronomical topics preferring the contact with astronomy to the unchartered seas revealed by this new alliance between computers and topology.

Two years ago at Salsjobaden in a conference on the Dynamics of Barred Spirals, George again broke open a new field (Contopoulos, 1997). His invariant dynamical spectra (described also in his contribution here) taught us how to measure and classify chaos, even complete chaos!

I have picked out a tiny fraction of George Contopoulos's work (1975) and mentioned things I learned from our direct contacts. He will no doubt deduce that I am not a very attentive pupil but it would be mean not to mention a lovely paper on the light distributions of elliptical galaxies (Contopoulos, 1956) because it is a beautiful work to which I constantly have to refer my astronomical colleagues!

The essence of this paper can be deduced by the following argument. Consider a spherical galaxy with any radial light profile. Now flatten its density distribution by linear contraction along any axis. This contraction can be resolved into one along and one perpendicular to the line of sight. The one along makes no difference while the one perpendicular flattens the circular distribution of observed light into one stratified on similar ellipses. If a further contraction is made along another axis we can apply the same argument again since ellipses contracted along any direction remain ellipses. So we arrive at George's beautiful theorem that if the density distribution of an elliptical galaxy is stratified on similar concentric ellipsoids then the light seen will be stratified on similar concentric ellipses whatever the orientation of the galaxy to the line of sight.

\section{Background to the Accretion Disc Theory of Quasars}

My own best work is "Galactic Nuclei as Collapsed Old Quasars" written in 1969. Then the discovery of quasars by Schmidt using Hazard's accurate position for one of Ryle's radio sources was still recent and quasars themselves were enigmatic objects more especially so because even the brightest 3C273 and 3C48 too did not seem to be associated with clusters of galaxies.

No-one then knew that Michell in his wonderfully percipient paper of 1784 had predicted both giant black holes and how they would be discovered! Even the name black hole only came into general use in 1970! In my 1969 paper I refer to "Schwarzschild throats". Laplace's translation of Michell's work into French (without attribution!) was not common reading among astronomers either.

Among the modern works on quasars as accretion discs, priority goes to Salpeter's fine 1964 letter to the Astrophysical Journal. Turning against the then common view 
that quasars were not associated with clusters of galaxies he worked out the consequences of a large black hole moving through a galaxy and accreting according to the Hoyle \& Lyttleton formula. He derived the power emitted per unit accretion rate by considering the binding energy of the last stable circular orbit and deduced a number of consequences of such black holes accreting as they wandered through the interstellar gas of a galactic disc. Five years elapsed before I wrote my paper. Originally unaware of Salpeter's (1964) note I luckily learned of it before the proofs came and so was able to add a sentence and a reference to his work. My aim was to show that the very small nuclei already known in the centres of galaxies were likely to be stars gathered around the giant-black-hole remnants of quasars. At the time, 1969, we already knew that the Optical Violently Variable or OVV quasars could change by a magnitude from one night to the next. Geoffrey Burbridge (1958) had been insistent that the giant radio sources needed $10^{61}$ ergs in fast electrons and magnetic field, while Ryle (1968) had emphasised that quasars would not be distinguished from such sources by radio measurements.

Now $10^{61}$ ergs weigh $\frac{1}{2} 10^{7} \mathrm{M}_{\odot}$. If one entertained the idea that these ergs came from nuclear energy then the $1 \%$ mass conversion efficiency of nuclear burning means that $10^{9} \mathrm{M}_{\odot}$ are needed. However putting $10^{9} \mathrm{M}_{\odot}$ within the light-variation-time length-scale of 10 light hours gives a gravitational binding energy of $10^{62} \mathrm{ergs}$ - on such a hypothesis $10^{62}$ ergs of gravitational energy would have been lost, all in order to burn $10^{9} \mathrm{M}_{\odot}$ of hydrogen into Helium and thereby get the mere $10^{61}$ ergs needed. This shows that in assuming nuclear power we nevertheless conclude that most of the energy comes from gravity. So the nuclear idea is not sensible and we should assume a preponderant gravity power and a somewhat smaller mass $\sim 10^{8} \mathrm{M}_{\odot}$. If conversion of mass into radiation is not $100 \%$ efficient quasars must leave behind massive remnants of $>10^{7}-10^{8} \mathrm{M}_{\odot}$ and because they have radiated their binding energy they have insufficient energy to re-expand. Since the masses are far beyond the Chandrasekhar limit there are no other final resting places other than giant black holes. Turning to the numbers of quasars derived by Sandage and estimating possible lifetimes, I deduced

$$
\begin{gathered}
\text { Number of clusters } \\
\text { of galaxies }
\end{gathered}<\begin{gathered}
\text { Number of dead } \\
\text { quasars }
\end{gathered}<\begin{gathered}
\text { Number of } \\
\text { galaxies }
\end{gathered}
$$

Thus the nearest dead quasar must be nearer than M87 and there may be as many dead quasars as there are massive galaxies. How could we hide dead quasars of $10^{8} \mathrm{M}_{\odot}$ when they still gravitate? They would naturally be centres of attraction for stars so it is natural to find such a body at the centre of an exceptionally dense region. Galactic Nuclei then became the obvious candidates so I looked at the Galaxy, the Magellanic Clouds, M31, M32, M81, M82, NGC4151, M87, etc. and estimated possible black hole masses from the 1969 data on their nuclei, many of which were due to pioneering work by Merle Walker, see Figure 1. I also drew on the accretion discs of my thesis and, finding the gaseous viscosity too low, I estimated a magnetic viscosity. This was based on the shearing of the disc causing magnetic reconnection and continual flaring above the disc. Indeed I found that the protons got most of the energy as they more readily achieved "runaway". Particle energies 


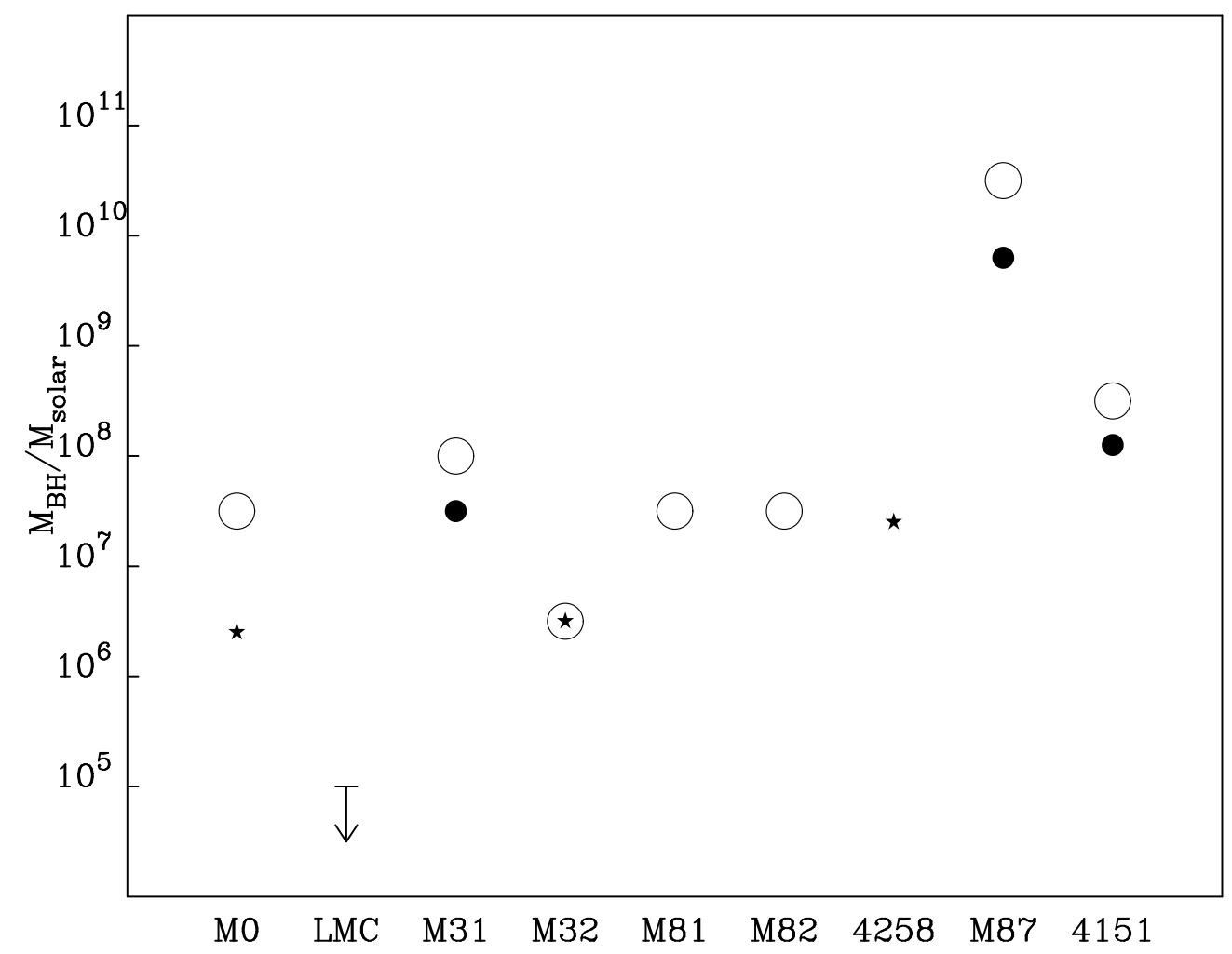

Figure 1: Comparison of my 1969 estimates of black hole masses $\bigcirc$ with modern determinations $\star$ and less precise modern estimates $\bullet$. Evidently the early estimates were somewhat over enthusiastic!

up to $10^{13} \mathrm{eV}$ were readily generated and hard emission would result when this hit the disc material. While the energy was primarily dissipated into such fast cosmic rays they would collide with the disc and heat it to temperatures $T \propto r^{-3 / 4}$ for $r \gg 2 G M_{0} / c^{2}$. Adding together such black body rings of emission, I got the disc spectrum $S_{\nu} \sim \nu^{1 / 3} \exp -\left(h \nu / k T_{\max }\right)$ where $T_{\max }$ the maximum temperature in Kelvin was $6.6 \times 10^{4} F_{-3}^{1 / 4} M_{7}^{-1 / 3}$; here $F_{-3}$ is the mass flux in units of $10^{-3} \mathrm{M}_{\odot} / \mathrm{yr}$ with $M_{7}$ the black hole's mass in units of $10^{7} \mathrm{M}_{\odot}$. I did not estimate how much hard emission would come from the initial collisions of the cosmic rays with the disk but a $10^{13} \mathrm{eV}$ cosmic ray is certainly capable of emitting hard $\gamma$ rays at its first few collisions. Even today, 29 years later, I think this model deserves more attention as a serious rival to the currently popular advection models.

The following year Jim Bardeen wrote a particularly fine paper which showed how accretion would spin up a Schwarzschild hole and after a finite mass was accreted leave it growing as a near-limiting Kerr hole of significantly greater efficiency. I gave a paper on these models at the 1970 Vatican Symposium on the nuclei of galaxies and in 1971 re-estimated the luminosity functions of quasars and mini quasars by developing the $\mathrm{C}^{-}$method. That year Ekers was stimulated to look with higher radio resolution at the Galactic Centre (Ekers \& Lynden-Bell, 1971) 
and I reviewed the then known data on it with Rees (Lynden-Bell \& Rees, 1971). A year or two later attention turned to lower mass black holes with the discovery of many X-ray binaries by the UHURU satellite. Papers by Pringle \& Rees (1972) and Shakura \& Sunyaev $(1973,1976)$ applied such ideas on a smaller scale and with Pringle I applied them (1974) to star formation both with and without magnetic fields. In 1978 I introduced the thick Kerr and Schwarzschild vortices in the hope of getting a more natural collimation mechanism than that of Blandford \& Rees (1974) but the very narrow jets are still inadequately understood.

\section{General Exact Solution to an Extraordinary N-body Problem}

I now return to the N-body problem and the little known fact that in Principia Newton (1687) solved an N-body problem in which every body attracts every other one and he solved it for all initial conditions!

His was the first of the class of extraordinary N-body problems which Ruth Lynden-Bell and I have been studying. Newton took the force between two bodies $i$ and $j$ to be $F=k m_{i} m_{j}\left(\mathbf{x}_{j}-\mathbf{x}_{i}\right)$. To get the total force in particle $i$ he summed over $j$ and since the $j=i$ term is zero we may sum over all $j$ to obtain

$$
\mathbf{F}_{i}=\sum_{j} \mathbf{F}_{i j}=k m_{i} M\left(\overline{\mathbf{x}}-\mathbf{x}_{i}\right)
$$

where $\overline{\mathbf{x}}$ is the position vector of the centre of mass which of course moves uniformly in a straight line and $M$ is the total mass of the system. Thus with this linear massweighted law, that Newton would never have ascribed to Hooke, the total force on the $i^{\text {th }}$ body is directed to the centre of mass and proportional to the distance from it. Therefore Newton found that each body describes a centred ellipse about the centre of mass which itself moves uniformly. This completes Newton's solution. In his case the potential energy is

$$
V=-\frac{1}{2} K \sum_{i<j} \sum_{j} m_{i} m_{j}\left(\mathbf{x}_{i}-\mathbf{x}_{j}\right)^{2}=-\frac{1}{2} k M \sum_{i} m_{i}\left(\mathbf{x}_{i}-\overline{\mathbf{x}}\right)^{2}=-\frac{1}{2} k M^{2} r^{2} .
$$

Generalising some work on statistical mechanics by Ruth Lynden-Bell we were led to consider the dynamics of N-body systems with the more general potential energy $V=V(r)$ where $r$ is given above. (cf. equation (2)). We define a mass weighted radius $\mathbf{r}$ in $3 N$ dimensions by

$$
\mathbf{r}=\left(\sqrt{\frac{m_{1}}{M}}\left(\mathbf{x}_{1}-\overline{\mathbf{x}}\right), \sqrt{\frac{m_{2}}{M}}\left(\mathbf{x}_{2}-\overline{\mathbf{x}}\right), \sqrt{\frac{m_{N}}{M}}\left(\mathbf{x}_{N}-\overline{\mathbf{x}}\right)\right),
$$

so the first 3 of the $N$ coordinates tell us where particle 1 is, the next 3 where particle 2 is, etc. Notice that $|\mathbf{r}|$ is the $r$ we defined previously. Equations of motion of the particles in centre of mass coordinates then lead directly to the equation

$$
M \ddot{\mathbf{r}}=-V^{\prime}(r) \hat{\mathbf{r}}
$$


where $\hat{\mathbf{r}}=\mathbf{r} / r$ is the unit radial vector in $3 N$ space.

One readily sees that

$$
r_{\alpha} \ddot{r}_{\beta}-r_{\beta} \ddot{r}_{\alpha}=0
$$

So

$$
r_{\alpha} \dot{r}_{\beta}-r_{\beta} \dot{r}_{\alpha}=L_{\alpha \beta}=-L_{\beta \alpha}=\text { const }
$$

Furthermore

$$
L^{2}=\frac{1}{2} L_{\alpha \beta} L_{\alpha \beta}=\frac{1}{2}\left(r_{\alpha} \dot{r}_{\beta}-r_{\beta} \dot{r}_{\alpha}\right)\left(r_{\alpha} \dot{r}_{\beta}-r_{\beta} \dot{r}_{\alpha}\right)=\left[r^{2}(\dot{\mathbf{r}})^{2}-(\mathbf{r} \cdot \dot{\mathbf{r}})^{2}\right]
$$

where $L^{2}$ is the constant defined by the first equality.

The energy in centre of mass coordinates is given therefore by

$$
\frac{1}{2} M \dot{\mathbf{r}}^{2}+V(r)=E=\frac{1}{2} M\left(\dot{r}^{2}+L^{2} r^{-3}\right)+V(r) .
$$

This determines $r(t)$ as a periodic function if $E<0$ so there is no violent relaxation in these systems and they vibrate eternally.

Differentiating (13) we find

$$
M\left(\ddot{r}-L^{2} r^{-3}\right)=-V^{\prime}(r) .
$$

This is the same equation of motion as that for the central distance to an object in planar motion which angular momentum $L$ about a centre of force with potential $V(r)$. It is natural to imagine such a planar orbit and to invent an angle $\phi$ such that $\phi=0$ at some pericentre and

$$
r^{2} \dot{\phi}=L,
$$

we may then imagine an orbit in two dimensional polar coordinates $r, \phi$ and following Newton we shall cling to the geometry by eliminating the time in favour of $\phi$. Now

$$
\ddot{\mathbf{r}}=\frac{d^{2}}{d t^{2}}(r \hat{\mathbf{r}})=\frac{d}{d t}\left(\dot{r} \hat{\mathbf{r}}+\frac{L}{r} \frac{d \hat{\mathbf{r}}}{d \phi}\right)=\ddot{r} \hat{\mathbf{r}}+\frac{L^{2}}{r^{3}} \frac{d^{2} \hat{\mathbf{r}}}{d \phi^{2}}
$$

where two terms in $\dot{r} L r^{-2} d \hat{\mathbf{r}} / d \phi$ cancel at the last step. Inserting this result into our equation of motion (9) and using (14), we deduce the wonderfully simple equation

$$
d^{2} \hat{\mathbf{r}} / d \phi^{2}+\hat{\mathbf{r}}=0,
$$

whose solution is

$$
\hat{\mathbf{r}}=\mathbf{A} \cos \phi+\mathbf{B} \sin \phi
$$

where $\mathbf{A}$ and $\mathbf{B}$ are constant $3 N$-vectors which obey $A^{2}=B^{2}=1$ and $\mathbf{A} \cdot \mathbf{B}=0$ in order that $\hat{\mathbf{r}}$ should be a unit vector for all $\phi$. Three further constraints on $\mathbf{A}$ and $\mathbf{B}$ follow from the fixed centre of mass. They are detailed in our paper but need not concern us here.

We now have the general solution, the centre of mass moves uniformly in a line and the particles pursue orbits about it of the form

$$
\mathbf{r}=r(\phi)(\mathbf{A} \cos \phi+\mathbf{B} \sin \phi),
$$


where $r(\phi)$ is the form of the two dimensional orbit governed by equations (13) and (15). These can be integrated explicitly for the Isochrone potential $V \propto k /(b+s)$, $s^{2}=r^{2}+b^{2}$ and for the Kepler and harmonic oscillator potentials. For the Kepler case $r(\phi)=\ell /(1+e \cos \phi)$ so the solution is of the pleasing form

$$
\mathbf{r}=\ell(1+e \cos \phi)^{-1}(\mathbf{A} \cos \phi+\mathbf{B} \sin \phi) .
$$

If we concentrate on the particle $i$, we find its orbit lies in the plane perpendicular to $\mathbf{A}_{i} \times \mathbf{B}_{i}$ where $i$ denotes the three components corresponding to particle $i$. Taking $x, y$ coordinates in that plane and eliminating $\phi$ we find that the orbit is quadratic. If $e$ were zero it would be a central ellipse, while if $\left|\mathbf{A}_{i}\right|$ and $\left|\mathbf{B}_{i}\right|$ are equal and orthogonal it gives a Keplerian eccentric ellipse. In the general bound case the ellipse has neither its centre nor its focus at the centre of mass $r=0$. These systems obey the equilibrium Virial theorem in the form $2 \mathcal{T}-r V^{\prime}=0$, so for the hyper-keplerian case $V \propto r^{-1}$ it takes the more familiar form $2 \mathcal{T}+V=0$.

One may work out the microcanonical statistical mechanics and find that $E=-\frac{3}{2}(N-2) k T$ so that the heat capacity $C=-\frac{3}{2}(N-2) k$ which is clearly negative as for other gravitating systems (Lynden-Bell \& Wood, 1968) and black holes. If $V$ takes the form

$$
V= \begin{cases}\infty & r<b \\ -k M^{2} / r & b<r<R \\ \infty & r>R\end{cases}
$$

corresponding to a gravitating system which cannot get too small or too big then a Canonical ensemble is possible and the negative specific heat region of the microcanonical ensemble is replaced by a giant first order phase transition as in our earlier model (Lynden-Bell \& Lynden-Bell, 1977).

\section{3b Generalisation}

We may extend these extraordinary N-body problems by taking $V$ to be of the more general form

$$
V=V_{0}(r)+r^{-2} V_{2}(\hat{\mathbf{r}})
$$

the only restriction on the second term being that it scales under expansion as $r^{-2}$. Those familiar with separable systems in 3 dimensions will know that for such potentials $\left(\frac{1}{2} m \mathbf{h}^{2}+V_{2}\right)$ is constant along an orbit where for that case $V_{2}=V_{2}(\theta, \phi)$ and $\mathbf{h}=\mathbf{r} \times \mathbf{v}$. The generalisation to $3 N$ dimensions is the first integral $\frac{1}{2} M L^{2}+V_{2}(\hat{\mathbf{r}})=\frac{1}{2} M \mathcal{L}^{2}$ say (note that due to the $V_{2}$ term, $\mathcal{L}^{2}$ does not have to be positive).

The energy equation now reads

$E=\frac{1}{2} M \dot{\mathbf{r}}^{2}+V_{0}+r^{-2} V_{2}=\frac{1}{2} M\left(\dot{r}^{2}+L^{2} r^{-2}\right)+V_{0}+r^{-2} V_{2}=\frac{1}{2} M\left(\dot{r}^{2}+\mathcal{L}^{2} r^{-2}\right)+V_{0}$

so the $r$ motion pulsates for ever as before. These systems show no violent relaxation in their breathing mode which pulsates (or evolves $E>0$ ) independently of the 
complication of the $\hat{\mathbf{r}}$ motion. Since $V_{2}(\hat{\mathbf{r}})$ is still free to choose, that motion can be as complicated as we like to make it. Defining a new time $\tau$ by $d / d \tau=1 / r^{2} d / d t$ the equations of motion for $\hat{\mathbf{r}}$ as a function of $\tau$ are totally independent of the $r$ motion, having a reduced Lagrangian system of their own in $\tau$-time. An interesting case to consider is the statistical mechanics of a "hard cone" gas in which $V_{2}$ is large and repulsive only in very small regions where two particles are nearly in the same direction as seen from the mass centre. This corresponds to the small hard sphere gas so beloved of textbooks. Carrying out that statistical mechanics, which is totally independent of any $r$ motion that may be going on, we obtain a new system at equilibrium in its $\hat{\mathbf{r}}$ coordinates but pulsating or evolving in $r$.

We have shown (Lynden-Bell \& Lynden-Bell, 1998) this equilibrium to be best described in terms of the peculiar velocity $\mathbf{v}_{i}$ relative to a "Hubble flow" $H\left(\mathbf{x}_{i}-\overline{\mathbf{x}}\right)$ where $H=\dot{r} / r$ that is

$$
\begin{aligned}
\mathbf{v}_{i} & =\dot{\mathbf{x}}_{i}-\dot{\overline{\mathbf{x}}}-H\left(\mathbf{x}_{i}-\overline{\mathbf{x}}\right) \\
f\left(\mathbf{v}_{i}, \mathbf{x}-\overline{\mathbf{x}}\right) & \propto \exp \left[-\left(\widetilde{\beta} r^{2} \frac{1}{2} m_{i} v_{i}^{2}\right)-\frac{\widetilde{\beta} r_{i}^{2}}{2 r^{2}}\right] .
\end{aligned}
$$

Thus the distribution is Maxwell-Boltzmann relative to be mean Hubble flow with a temperature proportional to $r^{-2}(t)$ and the profile is gaussian with a dispersion proportional to $r(t)$. It is notable that the 'equilibrium' of the $\hat{\mathbf{r}}$ coordinates is maintained throughout the pulsation just as the Planck distribution of cosmic black-body radiation in the Universe is maintained without interaction during the expansion of the Universe. Thus whether the relaxation to equilibrium of the angular coordinates is longer than or shorter than the pulsation time of $r$ is not relevant because 'equilibrium' once attained is maintained throughout the pulsation, it does not have to be recreated as each radius $r$ is attained. 


\section{References}

[1] AARSETH, S.J. 1974. Dynamical evolution of simulated star clusters. Astron. \& Astrophys. 35: 237.

[2] ANTONOV, V.A. 1962. Most probable phase distribution in spherical star systems and conditions for its existence. Originally Vest Leningrad Univ. 7: 135. Translation, 1995. IAU Symposium 113, 525.

[3] BARDEEN, J.M. 1970. Kerr metric black holes. Nature 226: 74.

[4] Betteweiser, E. \& D. SUGIMOTO. 1984. Post collapse evolution and gravothermal oscillation of globular clusters. Mon. Not. R. Astr. Soc. 208: 493.

[5] BLANDFORD, R.D. \& M.J. REES. 1974. A twin-exhaust model for double radio sources. Mon. Not. R. Astr. Soc. 169: 395.

[6] BURBIDGE, G.R. 1958. Possible sources of radio emission in clusters of galaxies. ApJ 128: 1.

[7] CAJORI, F. 1939. Newton's Principia. Motte's Translation Revised. University of California Press.

[8] CHANDRASEKHAR, S. 1995. Newton's Principia for the Common Reader. Oxford University Press.

[9] CONTOPOULOS, G. 1956. On the isophotes of ellipsoidal nebulae. Z.f. Astrophysik 39: 126 .

[10] CONTOPOULOS, G. 1960. A third integral of motion in a galaxy. Z.f. Astrophysik 49: 273.

[11] CONTOPOUlOS, G. 1965. Periodic and tube orbits. AJ 70: 526.

[12] CONTOPOUlOS, G. 1966. Recent Developments in Stellar Dynamics. In IAU Symposium No. 25, The Theory of Orbits in the Solar System and Stellar Systems. G. Contopoulos, Ed.

[13] CONTOPOUlOS, G. 1973. Topological Methods in Stellar Dynamics. In Dynamics of Stellar Systems, Saas Fée. Martinet \& Mayor, Eds. : pp52-87.

[14] CONTOPOULOS, G. 1975. Integrals of Motion. In IAU Symposium No. 64, Dynamics of Stellar Systems. Reidel.

[15] CONTOPOUlOS, G. 1997. Chaos in Barred Spiral Galaxies. In Nobel Symposium No. 98, Barred Galaxies and Grain Nuclear Activity. Springer.

[16] EDDINGTON, A.S. 1915. The dynamics of a stellar system III. Mon. Not. R. Astr. Soc. 76: 37 . 
[17] EKERS, R.D. \& D. LYNDEN-BELL. 1971. High resolution observations of the galactic centre at $5 \mathrm{GHz}$. ApJ Lett. 9: 189.

[18] HEGGIE, D. 1975. Binary evolution in stellar dynamics. Mon. Not. R. Astr. Soc. 173: 729 .

[19] HENON, M. 1961. Sur l'evolution dynamique des amas globulaires. Annales d'Astrophysique 24: 369.

[20] HENON, M. 1969. Numerical study of quadratic area preserving mappings. Q.J. App. Math. 27: 291.

[21] HENON, M. 1974. Integrals of the Toda Lattice. Phys. Rev. B. 9: 1921.

[22] JEANS, J.H. 1928. Astronomy \& Cosmology 337, Cambridge Univ. Press.

[23] LAPLACE, P.S. 1795. Le Système du Monde, Paris.

[24] LYNDEN-BELL, D. 1969. Galactic nuclei as collapsed old quasars. Nature 223: 690.

[25] LYNDEN-BELL, D. 1970. Formation and Evolution of Bright Black Holes. In Vatical Symposium on Activity in the Nuclei of Galaxies. D.J. O'Conell, Ed.

[26] LYNDEN-BELL, D. 1971. The $\mathrm{C}^{-}$method and N-galaxies as mini-quasars. Mon. Not. R. Astr. Soc. 155: 95 \& 119.

[27] LYNDEN-BELL, D. 1978. Gravity power. Physica Scripta 17: 185.

[28] LYNDEN-BELL, D. \& R.M. LYNDEN-BELL. 1977. On the negative specific heat paradox. Mon. Not. R. Astr. Soc. 181: 405.

[29] LYNDEN-BELL, D. \& R.M. LYNDEN-BELL. 1998. Exact general solutions to extraordinary N-body problems. Proc. R. Soc. (London) A.

[30] LYNDEN-BELL, D. \& M. NOURI-ZONOZ. 1998. Classical monopoles: Newton, NUT-space, gravomagnetic lensing and atomic spectra. Revs. Mod. Phys. 70: 427 .

[31] LYNDEN-BELL, D. \& J.E. PRINGLE. 1974. The evolution of viscous discs and the origin of the nebular variables. Mon. Not. R. Astr. Soc. 168: 603.

[32] LYNDEN-BELL, D. \& M.J. REES. 1971. On quasars dust and the galactic centre. Mon. Not. R. Astr. Soc. 152: 461.

[33] LYNDEN-BELL, D. \& R. WOOD. 1968. The gravo-thermal catastrophe in isothermal spheres and the onset of red-giant structures in stellar systems. Mon. Not. R. Astr. Soc. 138: 495.

[34] MICHELL, J. 1784. On the means of discovering the distance magnitude of the fixed stars etc. Phil. Trans. R. Soc. (London) 74: 35-57. 
[35] NEWTON, I. 1687. Principia. Royal Society, London.

[36] POINCARÉ, H. 1892-99. Les Methodes Nouvelles de la Méchanique Celeste, Paris. Translated History of Modern Physics \& Astronomy, 1993. Vol. 13. D.L. Goroff, Ed. : American Institute of Physics.

[37] PRINGLE, J.E. \& M.J. REES. 1972. Accretion disc models for compact X-ray sources. Astron. \& Astrophys. 21: 1.

[38] RYLE, M. 1968. Radio Astronomy and Quasars I. In IAU Highlights of Astronomy. L. Perek, Ed.

[39] SALPETER, E.E. 1964. Notes: Accretion of interstellar matter by massive objects. ApJ 140: 796.

[40] SHAKURA, N.I. \& R.A. SUNYAEV. 1973. Black holes in binary systems. Astron. \& Astrophys. 24: 337.

[41] SHAKURA, N.I. \& R.A. SUNYAEV. 1976. A theory of instability of disc accretion onto black holes, galactic nuclei, quasars. Mon. Not. R. Astr. Soc. 175: 613.

[42] THIRRING, W. 1972. Negative specific heat. Essays in Physics 4: 125.

[43] WHITTAKER, E.T. 1959. Analytical Dynamics, 4th Edn. Ch. 16. Cambridge University Press. 